\title{
ENEMA DE SULCRALFATO PODE PREVENIR COLITE EM SEGMENTOS COLÔNICOS SEM TRÂNSITO FECAL?
}

\author{
CAN SUCRALFATE ENEMA PREVENT COLITIS IN COLONIC SEGMENTS WITHOUT FECAL TRANSIT?
}

Roberta Laís Silva MENDONÇA ${ }^{\oplus}{ }^{\circ}$, Danilo Toshio KANNO ${ }^{1 \oplus}$, José Aires PEREIRA ${ }^{1 \oplus}$, Fabio Guilherme CAMPOS $^{2 \odot}$, Camila Morais Gonçalves da SILVA $^{3}{ }^{\circ}$, Bruna Zini de Paula FREITAS ${ }^{\circ}$, Carlos Augusto Real MARTINEZ ${ }^{1,4}$

\begin{abstract}
RESUMO - RACIONAL: O estresse oxidativo é um dos principais mecanismos associados à ruptura dos mecanismos de defesa que formam a barreira epitelial cólica e reduz o conteúdo tecidua das proteínas claudina-3 e ocludina principais constituintes das junções de oclusão intercelulares. O sucralfato, possui atividade antioxidante e tem sido usado para tratar diferentes formas de colite. OBJETIVO: Mensurar o conteúdo tecidual de claudina-3 e ocludina da mucosa do cólon sem trânsito fecal, submetido à intervenção com sucralfato. MÉTODO: Trinta e seis ratos foram submetidos à colostomia do cólon esquerdo e fístula mucosa distal. Os animais foram divididos em dois grupos de acordo com a eutanásia ser realizada duas ou quatro semanas após a intervenção. Cada grupo foi dividido em três subgrupos de acordo com o tipo de intervenção realizada diariamente: solução salina isolada; sucralfato a $1 \mathrm{~g} / \mathrm{kg} /$ dia ou sucralfato a $2 \mathrm{~g} / \mathrm{kg} / \mathrm{dia}$. A colite foi diagnosticada por análise histológica adotando escala de validação prévia. A expressão tecidual de ambas as proteínas foi identificada por imunoistoquímica. O conteúdo das proteínas foi quantificado por análise de imagem assistida por computador. RESULTADOS: O escore inflamatório foi maior nos segmentos cólicos sem trânsito fecal e os enemas com sucralfato reduziram o escore inflamatório nesses segmentos, principalmente nos animais submetidos à intervenção com sucralfato em maior concentração e por período mais longo de intervenção. Houve aumento no conteúdo tecidual das proteínas claudina-3 e ocludina, relacionado com a concentração de sucralfato. O conteúdo tecidual de ambas as proteínas não se modificou com a duração da intervenção. CONCLUSÃO: Enemas com sucralfato reduzem a inflamação e aumentam o conteúdo tecidual de claudina-3 e ocludina na mucosa cólica sem trânsito intestinal.
\end{abstract}

DESCRITORES: Colite. Claudina-3. Ocludina. Processamento de imagem assistida por computador. Sucralfato.

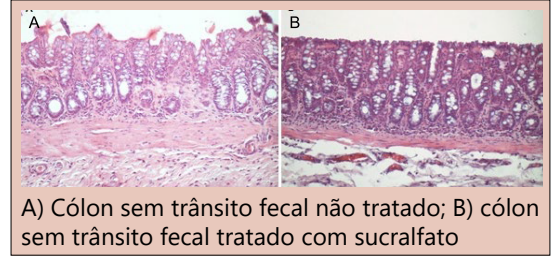

\section{Mensagem central}

A aplicação de enemas com sucralfato reduziu o processo inflamatório e aumentou o conteúdo das proteínas claudina-3 e ocludina em modelo experimental de colite de exclusão.

Perspectivas
Colite de exclusão é uma doença inflamatória
que ocorrenocólondesprovido de trânsito
fecal.Adoençacomprometea qualidade de
vida dosenfermos ea restauração do trânsito é
melhor opção terapêutica.Todavia, em muitos
doentes não pode ser realizada.Assim,estratégias
terapêuticasacessíveis, de baixo custo que
revertamo processo inflamatório e restabeleçam
a integridade epitelialsãobem-vindas. Neste
estudo mostramos que o uso deenemas com
sucralfato foi eficaz para o tratamento da
enfermidade.

Trabalho realizado no 'Laboratório de Investigação Médica, Programa de Pós-Graduação em Ciências da Saúde, Universidade São Francisco, Bragança Paulista, SP, Brasil; ${ }^{2}$ Departamento de Gastroenterologia, Faculdade de Medicina, Universidade de São Paulo, São Paulo, SP, Brasil; ${ }^{3}$ Departamento de Farmacologia, Centro Universitário da Uni Metrocamp, Campinas, SP, Brasil; ${ }^{4}$ Departamento de Cirurgia, Universidade Estadual de Campinas - Unicamp, Campinas, São Paulo - SP, Brasil.

Como citar esse artigo: Mendonça RLS, Kanno DT, Pereira JA, Campos FG, Silva CMG, Freitas BZP, Martinez CAR. Enema de sulcralfato pode prevenir colite em segmentos colônicos sem trânsito fecal ? ABCD Arq Bras Cir Dig. 2021;34(4):e1630. https://doi.org/10.1590/0102-672020210002e1630

\section{Correspondência:}

Carlos Augusto Real Martinez

Email: caomartinez@uol.com.br
Fonte de financiamento: Conselho Nacional de Desenvolvimento Científico e Tecnológico (CNPq) - Ministério de Ciência e Tecnologia do Brasil (Processo número: 303837/2018-7).

Conflito de interesses: Nenhum.

Recebido: 25/04/2021

Aceito: $18 / 08 / 2021$ 
INTRODUÇÃO

$\mathrm{O}$ epitélio colônico é a mais importante barreira de defesa do corpo humano'. Composta apenas por uma única camada de células especializadas forma barreira altamente dinâmica e seletiva que controla a absorção de fluidos e solutos, restringindo o acesso de patógenos aos tecidos subjacentes ${ }^{7,31}$. As células do epitélio colônico devem identificar e responder apropriadamente ao constante desafio imunológico do conteúdo luminal do cólon e, ao mesmo tempo, permitir a absorção de água, nutrientes e moléculas importantes para manter o metabolismo energético celular ${ }^{1}$. Esta eficiente função de barreira é obtida por uma série de junções intercelulares que incluem junções de oclusão apicais (JOs), junções aderentes subjacente, desmossomos e junções comunicantes, que medeiam a adesão intercelular e a comunicação entre células epiteliais adjacentes ${ }^{14}$. A camada de muco que recobre o epitélio colônico, a membrana citoplasmática das células que formam as glândulas cólicas, membrana basal, formam a barreira mecânica, enquanto as imunoglobulinas, citocinas e leucócitos a barreira imunológica e, também participam desse mecanismo de defesa ${ }^{14,22}$

As JOs representam o componente mais apical dos sistemas de junções intercelulares e fornecem forma eficiente de adesão célula-célula no epitélio colônico ${ }^{34}$. Elas conectam células adjacentes para determinar a permeabilidade paracelular controlada através do espaço intercelular lateral ${ }^{34}$. Cada vez mais importância se atribui as JOs nos mecanismos de proliferação celular, produção de muco, identificação de antígenos e bactérias patogênicas e produção de peptídeos antimicrobianos para garantir uma diferenciação celular imunológica eficaz ${ }^{27}$. As JOs são formadas por várias proteínas, como as da família das claudinas, occludina, tricelulina e molécula de adesão juncional ${ }^{16}$.

A inflamação da mucosa, conforme observada na doença inflamatória intestinal, compromete a barreira epitelial, resultando na exposição dos compartimentos estéreis do tecido da lâmina própria à antígenos luminais e micróbios que contribuem, ainda mais, para a resposta inflamatória e lesão da barreira epitelial 7,8,12. Estudo experimental mostrou que na colite de exclusão (CE), uma doença inflamatória que ocorre em segmentos do cólon desprovidos de trânsito fecal, as JOs ficam comprometidas ocasionando ruptura da barreira epitelial8,27. É possível que o aumento da produção de espécies reativas de oxigênio por células epiteliais privadas do fornecimento normal de ácidos graxos de cadeia curta, principal substrato energético para o metabolismo normal dessas células, seja um dos possíveis mecanismos relacionados à quebra das proteínas constituintes das $\mathrm{JOs}^{4,9,27}$. Essa possibilidade é reforçada pelos resultados de estudos mostrando que a aplicação de clisteres com várias substâncias com atividade antioxidante, ou soluções ricas em ácidos graxos de cadeia curta, podem melhorar o processo inflamatório da mucosa colônica exclusa de trânsito fecal e restabelecer os diferentes mecanismos de defesa que formam a barreira epitelial ${ }^{15,21,23,35}$.

O sucralfato (SCF) é um agente protetor celular usado há mais de três décadas no tratamento de úlceras pépticas duodenais e esofagite de refluxo ${ }^{29}$. A substância é um complexo de sacarose e sulfato-alumínio que, ao entrar em contato com a mucosa inflamada do trato gastrointestinal, adere fortemente a proteínas da superfície das ulcerações, principalmente albumina e fibrinogênio, formando um complexo estável e insolúvel, criando uma camada protetora que recobre e protege o epitélio danificado ${ }^{20,27}$. Estudos têm mostrado que o SCF pode ser usado com sucesso na retiteactínica ${ }^{20,28}$. Recentemente demonstrou-se que o uso do SCF em altas concentrações reduz a produção de espécies reativas de oxigênio e melhora a cicatrização da mucosa em modelos experimentais de $C E$, mostrando que a substância possui atividade antioxidante ${ }^{19}$. Enemas com SCF isolado ou associado à outras drogas têm demonstrado eficácia no tratamento de doenças inflamatórias intestinais e da $C E^{2,10,18,24}$. Contudo, do melhor do nosso conhecimento, nenhum estudo avaliou a eficácia da aplicação de clisteres contendo SCF, no conteúdo tecidual das proteínas claudina-3 e ocludina no epitélio colônico desprovido de trânsito fecal. É possível que o SCF, devido à sua atividade antioxidante, possa proteger as JOs da ação nociva das espécies reativas de oxigênio.

Assim, o objetivo do presente estudo foi quantificar o conteúdo tecidual das proteínas claudina-3 e ocludina na mucosa colônica desprovida de trânsito fecal submetida à aplicação diária de enemas contendo SCF em duas concentrações diferentes, por duas ou quatro semanas.

\section{MÉTODOS}

Este estudo foi realizado de acordo coma a Lei Federal Brasileira No.11.794é as orientações do Colégio Brasileiro de Experimentação Animal(COBEA). Este estudo experimental foi aprovado pelo Comitê de Ética no Uso de Animais em Pesquisa, Universidade São Francisco, Bragança Paulista - SP, Brasil (Processo №. 2211/07).

\section{Técnica cirúrgica: derivação do trânsito intestinal}

A metodologia cirúrgica utilizada para a indução da CE foi descrita anteriormente ${ }^{2,4}$. Resumidamente, 36 ratos foram submetidos a anestesia geralpor meio da administração intramuscular quetamina $0,1 \mathrm{ml} / 100 \mathrm{~g}$ exilazina $(20 \mathrm{mg} / \mathrm{ml}), 1: 1$ (v/v). A parede abdominal foi aberta por incisão mediana com $5 \mathrm{~cm}$ de extensãoe o cólon esquerdo foi seccionado $8 \mathrm{~cm}$ acima da margem anal e o segmento cranial exteriorizado como uma colostomia proximal. O segmento distaldo colón esquerdo seccionadofoi cateterizado com uma sonda de poliéstereirrigado com solução salinaaté que o efluente drenado pelo ânus do animalnão mais eliminasse resíduos fecais. Após a irrigação, o cateter foi removido, e o segmento distal do cólon exteriorizado como uma colostomia distal. A incisão abdominal foi fechada em dois planos de sutura. Durante o período pós-operatório, os ratos foram mantidos em gaiolas individuais, sem qualquer cuidado adicional com os estomas e incisão abdominal. A analgesia pós-operatória foi realizada pela administração de dipirona $(15 \mathrm{mg} / \mathrm{kg})$ diluída na água do bebedourooferecida diariamentenão sendo utilizados antibióticos. Após a operação, os animais foram mantidos nas gaiolas individuais por um período de seis semanas para o desenvolvimento daCE. Este mesmo período foi adotado em estudos anteriores ${ }^{30}$.

\section{Grupos experimentais}

Os 36 animais foram divididos em três grupos contendo 12 em cada. A intervenção com as soluções propostas foi iniciada seis semanas do pós-operatório da derivação do trânsito intestinal. O primeiro grupo recebeu enemas diários contendo SF 0,9\%.O segundo e terceiro grupos receberam enemas diários contendo SCF (EMS Sigma Pharma Ltda., Brasil) em duas diferentes concentrações $(1 \mathrm{~g} / \mathrm{kg} / \mathrm{diae} 2 \mathrm{~g} / \mathrm{kg} / \mathrm{dia}$, respectivamente). Em cada um dos grupos, seis animais foram sacrificados após duas semanas e outros seis após quatro semanas de intervenção.

\section{Coleta das amostras}

Após a conclusão do período de intervenção, os ratos foram novamente anestesiados como descrito anteriormente, $e$ a incisão mediana foi novamente aberta. Em ambos os grupos, foram retirados espécimes do cólon sem trânsito fecal submetidos à irrigação com SF 0,9\%ou SCF em ambas as concentrações. Foi retirado um espécime do cólon com $4,0 \mathrm{~cm}$ de comprimento. 
Para padronizar as análises histológicas, em todos os animais, esses segmentos do cólon sem fluxo fecal foram removidos sempre $0,5 \mathrm{~cm}$ acima da placa linfoide de Peyer. A seguir, os espécimes foram abertos através da borda antimesentérica fixados em um pedaço de cortiça e encaminhados para técnicas histológica e imuno-histoquímica. A eutanásia foi realizada por injeção intracardíaca de dose letal de tiopental.

\section{Análises histológicas}

Osespécimes de cólon sem trânsito fecal removido para as análises histológicas foram imersos em solução de formaldeído tamponado a $10 \%$ por 24 h, desidratados por exposição a concentrações crescentes de etanol, xileno e incluídos em parafina. Em seguida, seções do tecido emblocado,com $5 \mu \mathrm{m}$ de espessura. foram cortadas e montadas em uma lâmina de vidro. Posteriormente foram limpas, hidratadas e coradas com H\&E para avaliação da presença de colite e do grau de inflamação. As lâminas foram analisadas em microscópio óptico (Eclipse F-50, Nikon Inc., Osaka, Japão) com ampliação de 200x. As lâminas foram preparadas e analisadas por patologista que desconhecia os objetivos do estudo e que avaliou tanto as alteraçõeshistológicas identificadas pela técnica da $\mathrm{H} \& \mathrm{E}$ quanto o estudoimunoistoquímico (anti-claudina-3 e anti-ocludina).

Foram tiradas fotomicrografias utilizando uma câmera de captura de vídeo digital (DS-Fi-50; Nikon Inc., Osaka, Japão) acoplada ao corpo do microscópio. A presença de colite nos segmentos de cólon desprovidos de fluxo fecal foi confirmada considerando três parâmetros histológicos distintos: infiltração neutrofílica das camadas mucosa e submucosa, presença de erosões e ulceração epitelial e classificada em cruzes (+9) para cada variável considerada. A gravidade da inflamação na mucosa colônica desprovida de trânsito fecal foi estabelecida de acordo com uma escala de grau inflamatório previamente validada ${ }^{4}$.

\section{Coloração imunoistoquímica}

Para o estudo daimunoexpressão das proteínas claudina-3 e ocludina, utilizou-se metodologia padronizada adotada em outros estudos e obedecendo as recomendações técnicas dos fabricantes de cada um dos anticorpos primários ${ }^{16}$. Como anticorpo primário para pesquisa de claudina-3, foi empregado o anticorpo monoclonal anti-claudina-3 (Ref. E-3834, Lot. 110520, Spring Bioscience, Pleasanton, CA, EUA). O anticorpo primário anticlaudina-3 foi misturado numa diluição de 1:50 em soro de albumina bovina (1\%). O anticorpo monoclonal anti-ocludina (Ref. E-17464, Lot. 111207S, Spring Bioscience, Pleasanton, CA, EUA) foi diluído em 1:100 em soro de albumina bovina (1\%). As lâminas foram cobertas com aproximadamente $100 \mu \mathrm{L}$ dessas soluções e incubadas a $4^{\circ} \mathrm{C}$ por $24 \mathrm{~h}$. Após a exposição ao anticorpo primário, as lâminas foram lavadas com PBS e incubadas com o anticorpo secundário (Lote: H1011 HistofineCode: 414191N, Spring Bioscience, Pleasanton, CA, EUA). Em seguida, foram incubados com o complexo estreptavidina-biotina-peroxidase (ABC Staining System, Dako A/S, Glostrup, Dinamarca). A reação cromogênica foi desenvolvida com solução recém-preparada de DAB (tetrahidrocloreto de diaminobenzidina, $10 \mathrm{mg} \mathrm{em}$ $10 \mathrm{ml}$ de PBS). As lâminas foram lavadas e contrastadas com verde de metila por um minuto e, a seguir, lavadas novamente em água destilada. Em seguida, as lâminas foram desidratadas por imersão em concentrações crescentes de etanol seguida de xileno. Por fim, foram montadas, etiquetadas e mantidas na posição horizontal por $24 \mathrm{~h}$.

A imunocoloração foi considerada positiva quando se observava coloração acastanhada difusa com áreas de intensidade variável e distribuição homogênea nas membranas celular apical ou basolateral. Conforme recomendado pela bula de ambos os anticorpos primários usados, o controle negativo foi preparado sem a adição do anticorpo primário, enquanto o controle positivo para claudina-3 e ocludina foi realizado usando tecido de intestino delgado humano normal, que reconhecidamente apresenta positividade para ambas as proteínas.

Processamento de imagem assistido por computador

Oconteúdo tecidual de claudina-3e ocludina foi quantificado por meio de morfometria computadorizada e sempre realizado em campo focal no qual havia pelo menos três glândulas colônicas íntegras e contíguas. Essas imagens foram analisadas com o programa NIS-Elements 3.1 (Nikon Inc., Osaka, Japão). O programa transformava a intensidade da cor em número de pixels em cada campo histológico selecionado. Os valores dos pixels foram transformados em porcentagem da expressão da proteína pelos campos analisados (\%/campo). O valor final obtido em cada campo analisado nos segmentos do cólon foi a média dos valores encontrados na avaliação de três campos diferentes.

\section{Análise estatística}

A análise estatística foi realizada considerando nível de significância de $5 \%(p<0,05)$. Os dados obtidos de cada segmento de cólon analisado, em cada grupo experimental, foram expressos como valor médio com o respectivo erro padrão e analisados no programa estatístico Biostat 5.0. Para comparar o grau de escore inflamatório entre os diferentes grupos experimentais, foi utilizado o teste não paramétrico de MannWhitney. Para comparar o conteúdo de claudina-3 e ocludina entre os diferentes subgrupos experimentais, foi utilizado o teste $t$ de Student. Para analisar a variância do conteúdo de claudina-3 e ocludina nos tecidos entre os diferentes grupos experimentais, foi utilizada o teste ANOVA.

\section{RESULTADOS}

Na Figura 1 observa-se segmentos colônicos desprovidos de trânsito fecal em animais submetidos à intervenção com SF $0,9 \%$ e SCF na concentração de $2,0 \mathrm{~g} / \mathrm{kg} / \mathrm{dia}$. Animais submetidos à intervenção com SF 0,9\% apresentam maior dano epitelial quando comparados aos tratados com enemas contendo SCF na concentração de $2,0 \mathrm{~g} / \mathrm{kg} / \mathrm{dia}$.

Na Figura 2 observa-se o escore inflamatório, comparando segmentos colônicos sem trânsito fecal submetidos a intervenção com SF $0,9 \%$, SCF $1,0 \mathrm{~g} / \mathrm{kg} /$ diae $2,0 \mathrm{~g} / \mathrm{kg} / \mathrm{dia}$, porduas ou quatro semanas. $\mathrm{O}$ escore inflamatório reduziu apenas nos animais submetidos a intervenção quando utilizado maiores concentrações da droga e maior período de intervenção.

$\mathrm{Na}$ Figura 3 observa-se a expressão tecidual de clauidina-3, comparando segmentos colônicos sem trânsito fecal submetidos a intervenção com SF 0,9\% e SCF na concentração de 2,0 g/kg/dia, por quatro semanas.

A Figura 4 compara o conteúdo tecidual de claudina-3 em segmentos colônicos sem trânsito fecal submetidos à intervenção com SF 0,9\%, SCF 1,0g/kg/dia e 2,0 g/ kg/dia, por duas e quatro semanas. O conteúdo tecidual de claudina- 3 aumentou nos animais submetidos à intervenção com SCF independente da concentração utilizada ou do tempo de intervenção. Todavia, nos animais submetidos à intervenção com SCF em maiores concentraçõeso conteúdo tecidual de claudina-3 apresentou maior aumento.

Na Figura 5 observa-se a expressão tecidual da proteína ocludina, comparando segmentos colônicos sem trânsito fecal submetidos à intervenção com SF 0,9\% e SCF na concentração de $2.0 \mathrm{~g} / \mathrm{kg} / \mathrm{dia}$, por duas ou quatro semanas.

Na Figura 6 observa-se a comparação do conteúdo tecidual de ocludina nos segmentos colônicos sem trânsito fecal submetidos a intervenção com SF 0,9\%, SCF 1.0/kg/dia e $2.0 \mathrm{~g} / \mathrm{kg} / \mathrm{dia}$, por duas ou quatro semanas. O conteúdo de 


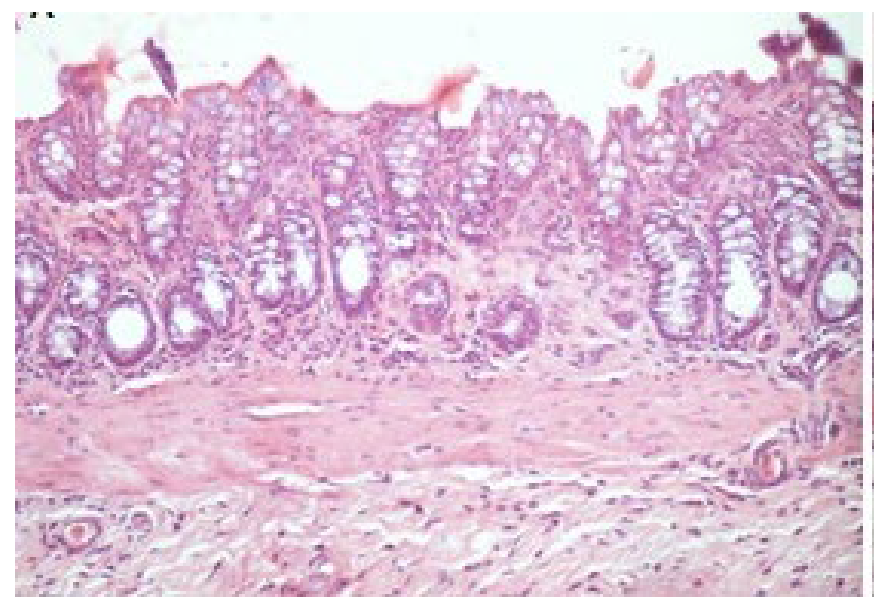

A

Figura 1 - A) Epitélio cólico sem trânsito fecal submetido a intervenção com SF 0,9\%por quatro semanas; B) epitélio colônico desprovido de trânsito fecal submetido à intervenção com SCF na concentração de 2,0 g/kg/dia

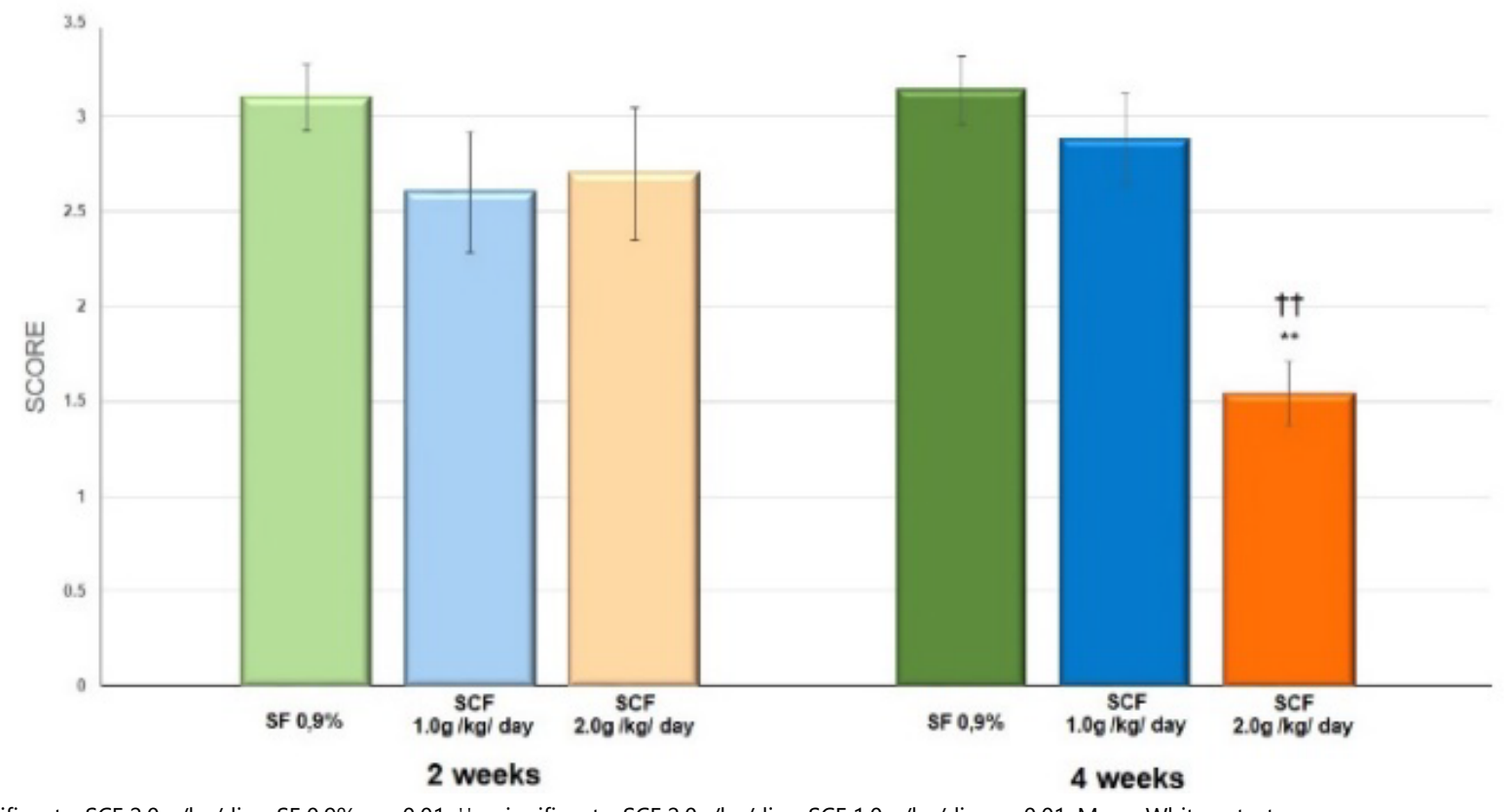

**=significante: SCF 2,0 g/kg/dia x SF 0,9\%; p< 0.01; +t =significante: SCF 2.0g/kg/dia x SCF 1,0 g/kg/dia, p <0.01. Mann-Whitney test.

Figura 2 - Valores médios do escore inflamatório encontrado nos animais submetidos a intervenção com SF 0,9\%, SCF 1,0g/ $\mathrm{kg} / \mathrm{dia}$ e $2,0 \mathrm{~g} / \mathrm{kg} /$ dia por duas ou quatro semanas.

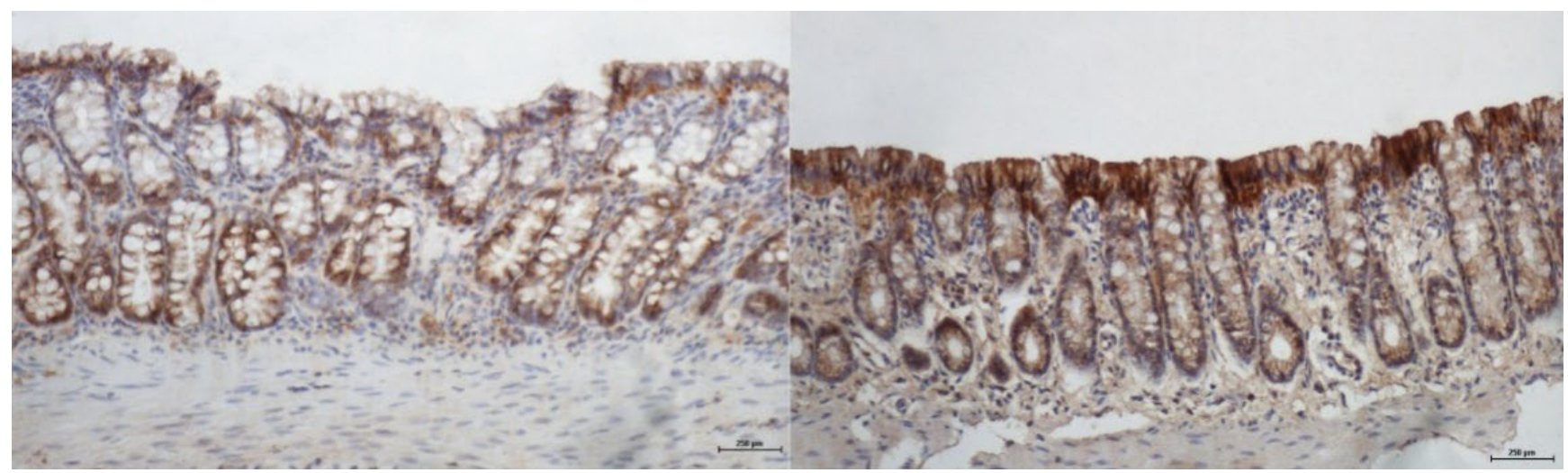

A

B

Figura 3 - A) Epitélio colônico sem trânsito fecal de animal submetido a intervenção com SF 0,9\% por quatro semanas, com redução da expressãode claudina-3 na superfície epitelial com a formação de úlcera (IH 200x); B) epitélio colônico sem trânsito fecal após intervenção com SCF 2,0 g/kg/dia, por quatro semanas com aumento da expressão da proteína claudina-3 na porção apical das glândulas cólicas (IH 200x). 


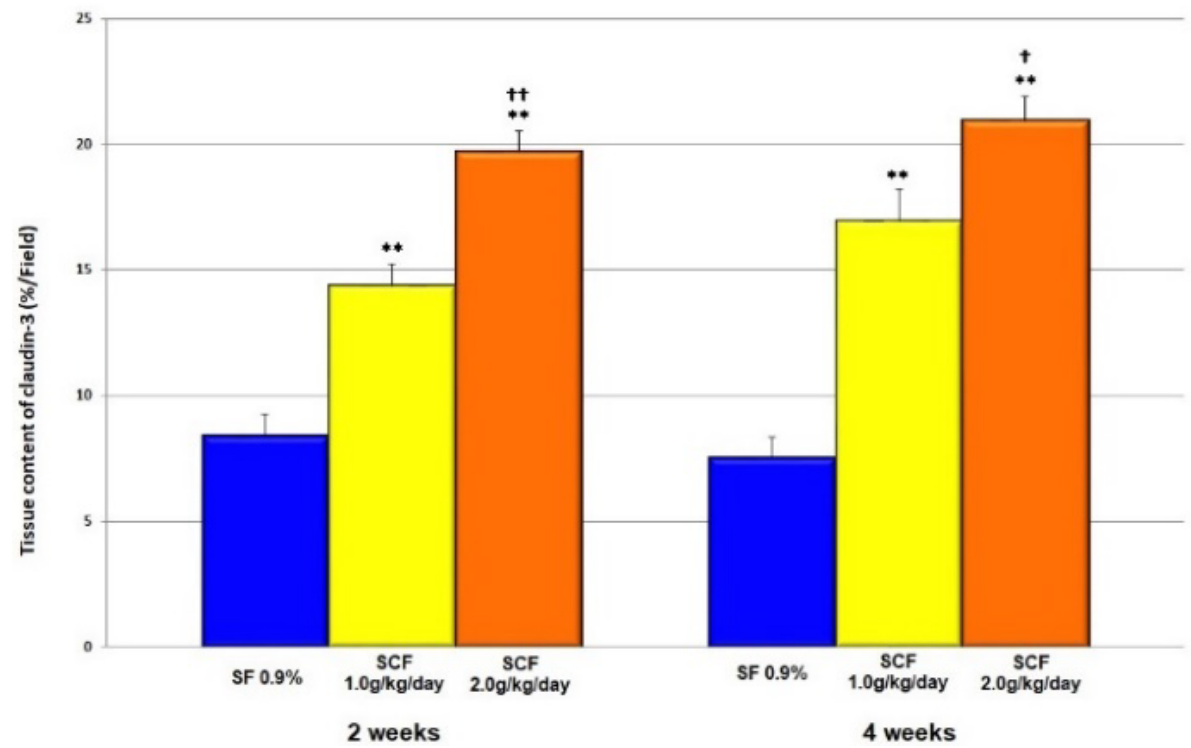

** = significante: SCF 1,0 g/kg/dia $\times$ SF 0,9\% e SCF 2,0 g/kg/dia $\times$ SF 0,9\% ( $p<0.0001) ;+$ significante; SCF 2,0 g/kg/dia $\times$ SCF 1,0 g/ $/ \mathrm{kg} / \mathrm{dia}(\mathrm{p}=0.01) ;++=$ significante: SCF $2,0 \mathrm{~g} / \mathrm{Kg} / \mathrm{dia} \times \mathrm{SCF} 1,0 \mathrm{~g} / \mathrm{kg} / \mathrm{dia}(\mathrm{p}=0.0003)$; Student $\mathrm{t}$ test.

Figura 4 - Valores médios para o conteúdo tecidual de claudina-3 encontrado nos animais submetidos à intervenção com SF $0,9 \%$, SCF $1,0 \mathrm{~g} / \mathrm{kg} / \mathrm{diae} 2.0 \mathrm{~g} / \mathrm{kg} / \mathrm{dia}$ porduas e quatro semanas.

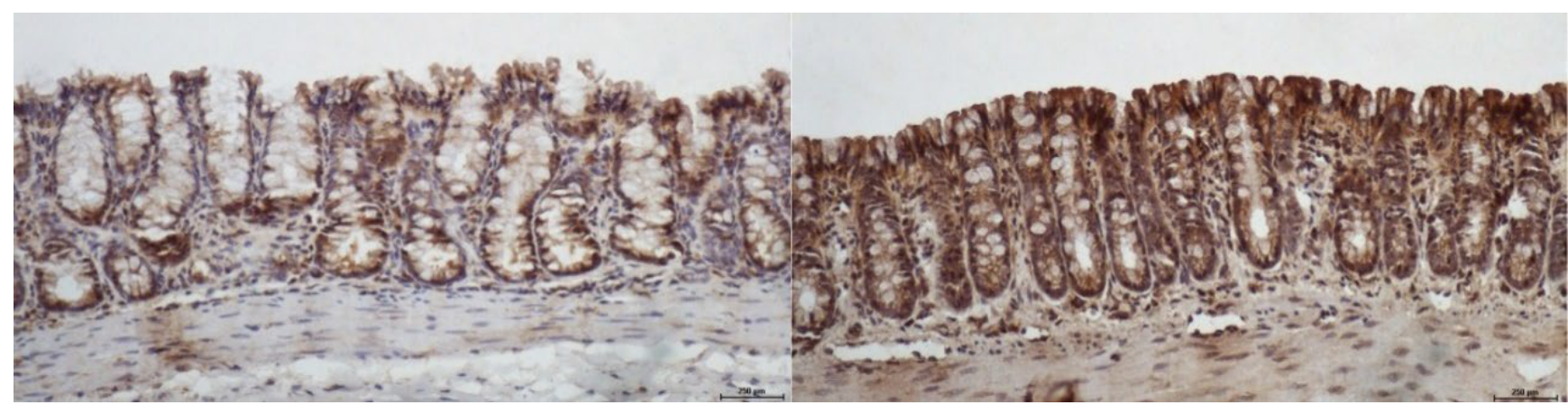

A

B

Figura 5 - A) Epitélio colônico sem trânsito fecal de animal submetido à intervenção com SF 0,9\%, por quatro semanas, com perda da expressão da proteína ocludina na superfície epitelial com a formação de várias ulcerações (IH 200x); B) epitélio colônico desprovido de trânsito fecal após intervenção com SCF 2,0 g/kg/dia por quatro semanas com aumento da expressão da proteína ocludina nas porções apical e basolateral das glândulas colônicas (IH 200x).

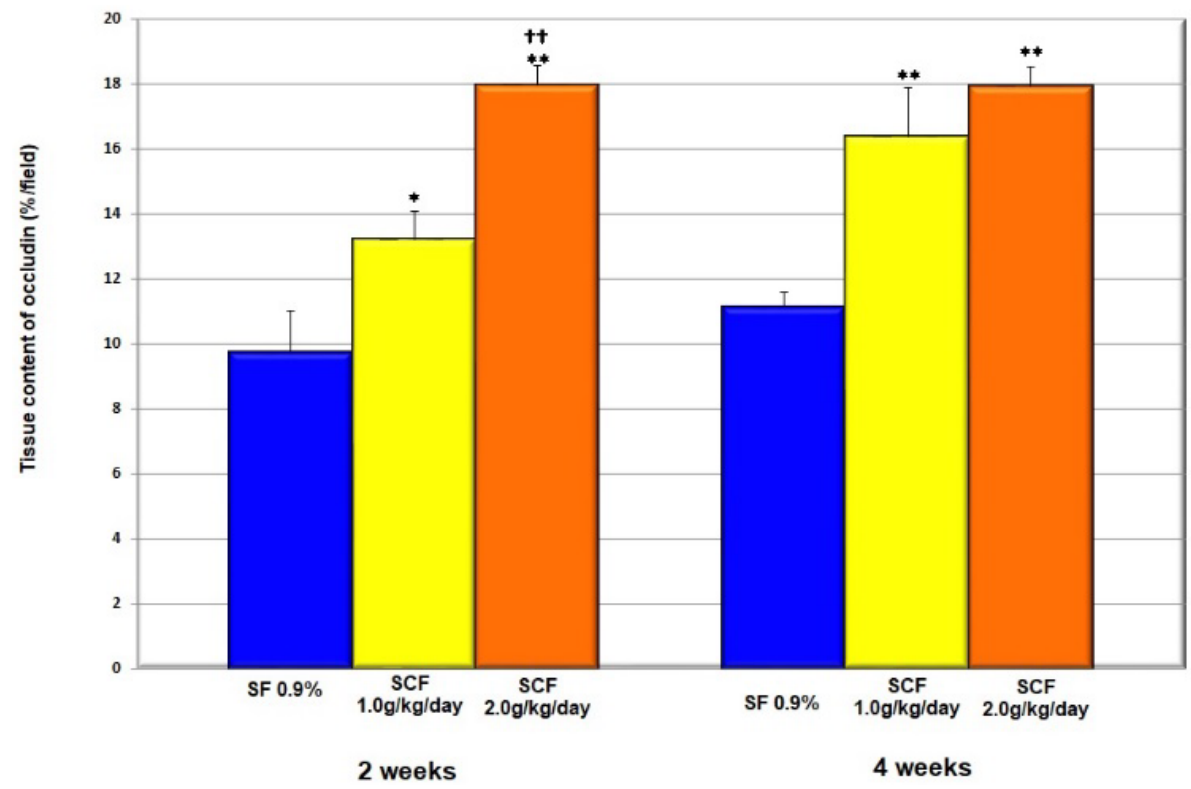

${ }^{* *}=$ significante: SCF 1,0 g/kg/dia $\times S F$ 0,9\%, and SCF 2,0 g/kg/dia $\times$ SF 0,9\% ( $p<0.0001$ ); $+t=s i g n i f i c a n t e: ~ S C F ~ 2,0 \mathrm{~g} / \mathrm{kg} / \mathrm{dia} \times S C F ~ 1,0 \mathrm{~g} / \mathrm{kg} / \mathrm{dia}$ ( $p=0.0003$ ) Student t test.

Figura 6 - Média dos valores teciduais do conteúdo de ocludina encontrado nos animais submetidos à intervenção com SF $0,9 \%$, SCF $1.0 \mathrm{~g} / \mathrm{kg} / \mathrm{dia}$ e $2.0 \mathrm{~g} / \mathrm{kg} /$ dia por duas e quatro semanas. 
ocludina aumentou nos animais submetidos a intervenção com SCF independente da concentração ou do tempo de intervenção. Não houve variação no conteúdo das proteínas claudina-3 e ocludina relacionadas ao tempo de intervenção (duas ou quatro semanas), nos animais submetidos à intervenção com SF $0,9 \%$, SCF $1,0 \mathrm{~g} / \mathrm{kg} / \mathrm{dia}$ ou SCF 2,0 g/ $\mathrm{kg} /$ dia.

\section{DISCUSSÃO}

SCFé o sal formado pelo dissacarídeo sacarose octassulfatado associado ao hidróxido de polialumínio ${ }^{13,33}$. A substância é considerada um agente citoprotetor e foi inicialmente utilizada para prevenir ou tratar doenças do trato digestivo superior, representadas principalmente pelaúlcera péptica, úlceras de estresse e erosões agudas da mucosa gástrica ${ }^{29,33}$. Posteriormente, devido sua grande capacidade de aderir a erosões do epitélio inflamado, o SCF também se mostrou eficaz para o tratamento de pacientes com proctite induzida por radiação e nos doentes com doença inflamatória intestinal, principalmente a retite erosiva distal $3,10,13,28$. Desde então, uma série de autores publicou os resultados do uso do SCF para o tratamento de diferentes doenças colorretais que evoluem com inflamação, incluindo colite ulcerativa e proctite por radiação ${ }^{25,26}$. Porém, revisando a literatura, nenhum estudo clínico avaliou a eficácia do SCF em pacientes com CE e, apenas nosso grupo, vem estudando a eficácia do SCF em um modelo experimental de $\mathrm{CE}^{2,6,18,24}$. Esses estudos mostraram que a aplicação diária de enemas com SCF reduzem o infiltrado inflamatório e o dano oxidativo na mucosa colônica desprovida de trânsito fecal, aumentam o conteúdo tecidual de vários tipos de mucinas que recobrem a mucosa, além de melhorar a cicatrização do epitélio colônico inflamado 2,18,19,24. Provavelmente, todos esses resultados estão relacionados à capacidade do SCF em estimular a produção de muco pelas células epiteliais da mucosa gastrointestinal, de aumentar a síntese do fator de crescimento epitelial estimulando a cicatrização epitelial e, principalmente, por sua ação antioxidante e anti-inflamatória ${ }^{19,29}$

O epitélio colônico atua como uma barreira morfológica e funcional, pois apesar de possuir permeabilidade seletiva garante a proteção contra a invasão de agentes nocivos presentes na luz intestinal ${ }^{4,31,32,34}$. Isso é alcançado por meio de múltiplos mecanismos de defesa que envolvem vários tipos de células epiteliais e não epiteliais - que atuam de forma integrada para construir barreiras protetoras nas mucosas ${ }^{25,30,31}$. Um dos mais importantes mecanismos de defesa érepresentado pelos sistemas de junções intercelulares, particularmente pelas JOs. Estudos em modelos experimentais de colite induzida e em pacientes com doença inflamatória intestinal têm demonstrado que a quebra das junções intercelulares é um evento precoce na etiopatogenia da doença ${ }^{8,11,14}$. O estresse oxidativo tem se mostrado um dos principais mecanismos envolvidos na quebra desses sistemas de junção intercelular ${ }^{9,12}$. Estudos mostraram que essas junções estão comprometidas em diferentes formas de colite e que apenas a deficiência de ácidos graxos de cadeia curta já pode causar ruptura das junções intercelulares ${ }^{32}$. A integridade das junções intercelulares já foi estudada em modelo experimental de $C^{16,17}$. Nestes estudos, foi medido o conteúdo tecidual das principais proteínas que formam asJOs (claudina- 3 e ocludina) comparando os segmentos do cólon providos e desprovidos detrânsito fecal. Verificou-se que ocorria redução acentuada do conteúdo de ambas as proteínas nas células das glândulas da mucosa cólica desprovida de trânsito intestinal ${ }^{16}$. Essa redução mostrou-se mais acentuada no conteúdo de claudina-3, principal proteína que constitui as JOs da mucosa colônica ${ }^{16}$. A redução no conteúdo tecidual de ambas as proteínas presentes nas JOs foi inversamente relacionada aos níveis de estresse oxidativo e ao agravamento da inflamação tecidual ${ }^{16}$. A aplicação de enemas contendo substâncias com destacada atividade antioxidantes, como extrato oleoso de curcumina, aumenta o conteúdo tecidual de claudina-3 e ocludina na mucosa colônica desprovida de trânsito intestinal ${ }^{17}$.

Quando se considera que as espécies reativas de oxigênio podem ocasionar quebra das proteínas presentes nas JOs em modelos experimentais de CE e que o SCF, além de possuir propriedades antioxidantes, pode proteger o epitélio intestinal por aumentar a produção de muco e favorecer a cicatrização epitelial, torna-se interessante avaliar a eficácia da substância na preservação das JOs em um modelo $C^{2,4,5,6,6,2,24}$. Os resultados deste estudo mostram que o conteúdo tecidual de ambas as proteínas aumentou nos segmentos colônicos desprovidos de trânsito fecal submetidos à intervenção com SCF, independente da concentração utilizada e do tempo de intervenção adotado. No entanto, quando o SCF foi usado na concentração de $2,0 \mathrm{~g} / \mathrm{kg} / \mathrm{dia}$, a manutenção do conteúdo tecidual de ambas as proteínas foi mais significativa. Nesta concentração, a pontuação do escore inflamatório também reduziu significativamente, confirmando as propriedades anti-inflamatórias da substância. Estudos anteriores também mostraram que a intervenção com SCF aumenta o conteúdo tecidual de mucinas neutras, mucinas ácidas totais, sulfomucinas, sialomucinas e MUC-2, relacionada a redução da intensidade inflamatória ${ }^{2,45,5,6,18,22,24}$. É provável que a ação antioxidante e anti-inflamatória do SCF, demonstrada em estudos anteriores, possa ser o principal mecanismo de proteção das $\mathrm{JOs}^{2,5}$.

Os resultados do presente estudo sugerem que o SCF pode ser uma estratégia terapêutica útil para o tratamento da CE. Por ser um medicamento de baixo custo e boa disponibilidade, é possível que seja utilizado em pacientes com $C E$, onde não se vislumbra a possibilidade de restabelecimento do trânsito fecal. No entanto, estudos em humanos, com maior número de pacientes e com maior tempo de seguimento ainda são necessários para confirmar essas perspectivas.

\section{CONCLUSÃO}

Enemas com SCF reduzem a inflamação e aumentam o conteúdo tecidual de claudina-3 e ocludina em segmentos colônicos desprovidos de trânsito fecal em modelo experimental de CD.

\section{REFERENCES}

1. Aukoetter MG, Nava $P$, Nusrat $A$. Role of the intestinal barrier in inflammatory bowel disease. World J Gastroenterol.2008;14(3):4017. doi.org/10.3748/WJG.14.401.

2. Bonassa CE, Pereira JA, Campos FG, Rodrigues MR, Sato DT, Chaim FD, Martinez CA. Tissue content of sulfomucins and sialomucins in the colonic mucosa, without fecal stream, undergoing daily intervention with sucralfate. Acta Cir Bras. 2015;30(5):328-38. doi. 10.1590/S0102-865020150050000004.

3. Carling I, Kagevi I, Borvall E. Sucralfate enema (SUC)--effective in IBD? Endoscopy. 1986;18(3):115. doi: 10.1055/s-2007-1018347.

4. Castilho TJC, Almeida GHDR, Mello EVSL, Campos ACL. Effect of supplementation with probiotics on colonic anastomoses in rats: morphological and tensiometric study. Arq Bras Cir Dig. 2021;33(4):e1550. doi: 10.1590/0102-672020200004e1550.

5. Chaim FM, Sato DT, Rodrigues MR, Dias AM, Silveira Júnior PP, Pereira JA, Martinez CA. Evaluation of the application of enemas containing sucralfate in tissue content of neutral and acid mucins in experimental model of diversion colitis. Acta Cir Bras. 2014; 29(9):544-52. doi.10.1590/S0102-8650201400150001. 
6. Fernandez OOA, Pereira JA, Campos FG, Araya CM, Marinho GE, Novo RS, Oliveira TS, Franceschi YT, Martinez CAR.Evaluation of enemas containing sucralfate in tissue content of MUC-2 protein in experimental model of diversion colitis. Arq Bras Cir Dig. 2018;31(3):e1391. doi. 10.1590/0102-672020180001e1391.

7. Garcia-Hernandez V, Quiros M, NusratA.Intestinal epithelial claudins: expression and regulation in homeostasis and inflammation. Ann N Y Acad Sci. 2017;1397(1):66-79. doi. 10.1111/nyas13360.

8. Gassler N, Rohr C, Schneider A, KartenbeckJ, Bach A, Obermüller N, Otto HF, Autschbach F. Inflammatory bowel disease is associated with changes ofenterocyticjunctions. Am JPhysiol Gastrointest Liver Physiol.2001;281(1):G216-28. doi:10.1152/ajpgi.2001.281.1.G216.

9. HaidariM.Zhang W, WakameK. Disruption of endothelial adherens junction by invasive breast cancer cells is mediated by reactive oxygen species and is attenuated by AHCC. LifeSci.2013;93(25-26): 994-1003. doi. 10.1016/j.lfs.2013.10.027.

10. Ispas-Szabo P, Friciu MM, Nguyen P, Dumoulin Y, Mateescu MA. Novelself-assembledmesalamine-sucralfatecomplexes:preparation, characterization, and formulation aspects. Drug Dev Ind Pharm. 2016;42(7):1183-93. doi. 10.3109/03639045.2015.1118493.

11. Iwaya $H$, Maeta $K$, Hara $H$, Ishizuka $S$. Mucosal permeability is an intrinsic factor in susceptibility to dextran sulfate sodium-induced colitis in rats. Exp Biol Med (Maywood). 2012;237(4):451-61. doi. 10.1258/ebm.2011.011269.

12. John LJ; Fromm M, Schulzke JD. Epithelial barriers in intestinal inflammation. Antioxid Redox Signal. 2011;15(5):1225 70. doi. 10.1089/ars.2011.3892.

13. KochharR, Patel F, DharA, SharmaSC, AyyagariS, Aggarwal R, Goenka MK, Gupta BD, Mehta SK. Radiation-induced proctosigmoiditis. Prospective, randomized, double-blind controlled trial of oral sulfasalazine plus rectal steroids versus rectal sucralfate. Dig Dis Sci. 1991;36(1):103-7. doi.10.1007/bf01300096.

14. Kucharzik T, Walsh SV, Chen J, Parkos CA, Nusrat A. Neutrophil transmigration in inflammatory bowel disease is associated with differential expression of epithelial intercellularjunction proteins. Am JPathol.2001;159(6):2001-9..doi..10.1016/S0002-9440(10)63051-9.

15. Luceri C, Femia AP, Fazi M, Di Martino C, Zolfanelli F, Dolara P, Tonelli F. Effect of butyrate enemas on gene expression profiles and endoscopic/ histopathological scores of diverted colorectal mucosa: A randomized trial. Dig Liver Dis. 2016;48(1):27-33. doi. 10.1016/j.dld.2015.09.005.

16. Martinez CA, de Campos FG, de Carvalho VR, de Castro Ferreira C, Rodrigues MR, Sato DT, Pereira JA. Claudin-3 and occludin tissue content in the glands of colonic mucosa with and without a fecal stream. J Mol Histol. 2015; 46(2):183-94. doi. 10.1007/ s10735-015-9610-y.

17. Martinez CA, Kadri CJ, Kanno DT, Alves AJ Júnior, Coy CS, Pereira JA. Claudin-3 and occludin content in the glands of colonic mucosa devoid from fecal stream submitted to topical intervention with oil extract of Curcuma longa. Acta Cir Bras. 2017;32(1):65-73. doi. 10.1590/s0102-865020170108.

18. Martinez CAR, Rodrigues MR, Sato DT, Silva CMG, Kanno DT, Mendonça RLS, Pereira JA. Evaluation of the anti-inflammatory and antioxidant effects of the sucralfate in diversion colitis. J. Coloproctol.2015;35(2):90-9. https://doi.org/10.1016/j.jcol.2015.02.007

19. Masuelli L, Tumino G, Turriziani M, Modesti A, Bei R. Topical use of sucralfate in epithelial wound healing: clinical evidence and molecular mechanisms of action. Recent Pat Inflamm Allergy Drug Discov. 2010;4(1):25-36. doi. 10.2174/187221310789895649.

20. Matsuu-Matsuyama M, Shichijo K, Okaichi K, Ishii K, Wen CY, Fukuda E, Nakayama T, Nakashima M, Okumura Y, Sekine I.
Sucralfate protects intestinal epithelial cells from radiation-induced apoptosis in rats. J Radiat Res. 2006;47(1):1-8. doi. 10.1269/jrr.47.1.

21. Nassri CGG, Nassri $A B$, Favero $E$, Rotta $C M$, Martinez CAR, Margarido NF.Influence of irrigation of nutritional solutions in the colon excluded of fecal stream. Experimental study in rats. Rev Bras Coloproctol. 2008; 28(3): 306-14. doi.org/10.1590/ S0101-98802008000300006.

22. Nonose R, Spadari AP, Priolli DG, Máximo FP, Pereira JA, Martinez $C A$. Tissue quantification of neutral and acid mucins in the mucosa of the colon with and without fecal stream in rats. Acta Cir Bras. 2009;24(4):267-275. doi.org/10.1590/S0102-86502009000400005.

23. Pacheco RG, Esposito CC, Müller LC, Castelo-Branco MT, Quintella LP, Chagas VL, de Souza HS, Schanaider A. Use of butyrate or glutamine in enema solution reduces inflammation and fibrosis in experimental diversion colitis. World J Gastroenterol. 2012;18(32):4278-87. doi.org/10.3748/wjg.v18.i32.4278

24. Pereira JA, Rodrigues MR, Sato DT, Silveira Júnior PP, Dias AM, Silva CMG, Martinez, CAR. Evaluation of sucralfate enema in experimental diversion colitis. J. Coloproctol. 2013; 33(4):182-90. doi.org/10.1016/j.jcol.2013.08.005

25. Porouhan P, Farshchian N, Dayani M. Management of radiationinduced proctitis. J Family Prim Med Care. 2019; 8(7):2173-2178. doi. 10.4103/jfmpc.jfmpc_333_19.

26. RileySA, Gupta I, ManiV.Acomparison of sucralfateand prednisolone enemas in the treatment of active distal ulcerative colitis. Scand J Gastroenterol. 1989;24(8):1014-8. doi. 10.3109/00365528909089249.

27. Saitou M, Furuse $M$, Sasaki $H$, Schulzke JD, Fromm M, Takano $H$, Noda T, Tsukita S. Complex phenotype of mice lacking occludin, a componentoftightjunctionstrands. Mol BiolCell.2000;11(12):4131-42. doi: $10.1091 / \mathrm{mbc}$.11.12.4131.

28. Sarin A, Safar B. Management of radiation proctitis. Gastroenterol Clin North Am. 2013;42(4):913-25. doi.org/10.1016/j.gtc.2013.08.004

29. Slomiany BL, Piotrowski J, Okazaki K, Grzelinska E, Slomiany A. Nature of the enhancement of the protective qualities of gastric mucus by sucralfate. Digestion. 1989; 44(4): 222-231. doi. org/10.1159/000199915

30. Sousa, MV, Priolli DG, Portes AV, Cardinalli IA, Pereira JA, Martinez CA. Evaluation by computerized morphometry of histopathological alterations of the colon wall in segments with and without intestinal transit in rats. Acta Cir Bras. 2008; 23(5):417-24. doi. 10.1590/ S0102-86502008000500005.

31. Thoo L, Noti M, Krebs P. Keep calm: the intestinal barrier at the interface of peace and war. Cell Death Dis. 2019;10(11):849. doi. org/10.1038/s41419-019-2086-z

32. Vivinus-NébotM, Frin-Mathy G, BziouecheH, Dainese R, Bernard G, Anty R, Filippi J, Saint-Paul MC, Tulic MK, Verhasselt V, Hébuterne $X$, Piche $T$. Functional bowel symptoms in quiescent inflammatory bowel diseases: role of epithelial barrier disruption and low-grade inflammation. Gut, 2014;63(5):744-52. doi.org/10.1136/gutjnl2012-304066.

33. Volkin DB, Verticelli AM, Marfia KE, Burke CJ, Mach $\mathrm{H}$, Middaugh CR. Sucralfate, and soluble sucrose octasulfate bind and stabilize acidic fibroblast growth factor. Biochim Biophys Acta.1993; 1203(1):18-26. doi.org/10.1016/0167-4838(93)90031-I.

34. Zihni C, Mills C, Matter K, Balda MS. Tight junctions: from simple barriers to multifunctional molecular gates. Nat Rev Mol Cell Biol. 2016;17(9):564-80. doi.org/10.1038/nrm.2016.80.

35. Zundler S, Dietz L, Matzel KE, Geppert Cl, Becker E, Rath T, Neurath MF, Atreya R. Successful Long-term Treatment of diversion colitis with topical coconut oil application. Am J Gastroenterol. 2018;113(12):1908-10. doi.org/10.1038/s41395-018-0394-z 